\title{
Análise dos estoques de carbono no solo sob diferentes coberturas vegetais no Brasil (revisão)
}

\author{
Josué Luiz Marinho Junior ${ }^{\mathrm{a}^{*}} \oplus$, Dayane de Souza Lima ${ }^{\mathrm{a}} \oplus$, João Lucas Aires Dias ${ }^{\mathrm{a}} \oplus$, \\ Renisson Neponuceno de Araújo Filho ${ }^{a}$ (C) \\ a Universidade Federal do Tocantins, Brasil \\ *Autor correspondente (josue.marinho@ hotmail.com)
}

\section{N F O}

\section{Keyworks}

anthropic actions

management and land

use

brazilian ecosystems

\section{Palavras-chave} ações antrópicas manejo e uso do solo ecossistemas brasileiros

\begin{abstract}
A B S T R A C T
Analysis of soil carbon stocks under different vegetation cover in Brazil (review)

Changes in vegetation cover of soil, cause changes in its structure and can impact the soil carbon stocks. The present study seeks to evaluate current publications on soil carbon stocks under different vegetation covers published in recent years and to carry out a critical and systemic assessment of subject, considering the methodologies used in each work and the different types of soil management and use in several regions of Brazil. For data collection, articles published in the last ten years (2010-2019) used in databases of scientific articles SciELO and ScienceDirect over a period of twelve days were used as an inclusion criterion, with the theme: "estoques de carbono no solo" or "soil carbon stocks", from which 1,186 articles were selected, 35 articles that most correlated with the theme. After the survey of articles, we sought to characterize the Brazilian ecosystems and summarize the main published data on carbon stocks in ecosystems and subsequently the stock estimates in environments under changing land use. The results showed that the different vegetation coverings present different, however positive capacity to store carbon in soil in Brazil and that variables such as handling, texture, physical, chemical and biological attributes are significant factors and can and directly influence the results.
\end{abstract}

\section{R E S U M O}

A mudança na cobertura vegetal do solo, causam alterações na sua estrutura e podem impactar nos estoques de carbono nos solos. O presente estudo busca avaliar publicações atuais sobre os estoques de carbono do solo sob diferentes coberturas vegetais publicados nos últimos anos e realizar uma avaliação crítica e sistêmica do assunto, considerando as metodologias utilizadas em cada trabalho e os diferentes tipos de manejo e uso dos solos em várias regiões do Brasil. Para a coleta de dados foi utilizado como critério de inclusão, artigos publicados nos últimos dez anos (2010-2019) pesquisados nas bases de dados de artigos ciêntíficos SciELO e ScienceDirect em um período de doze dias, com o tema: "estoques de carbono no solo" ou "soil carbon stocks", onde, de 1.186 artigos foram selecionados 35 artigos que mais se correlacionavam com o tema. Feito o levantamento dos artigos buscou-se caracterizar os ecossistemas brasileiros e resumir os principais dados publicados sobre os estoques de carbono nos ecossistemas e posteriormente as estimativas dos estoques em ambientes sob mudança uso do solo. Os resultados apontaram que as diferentes coberturas vegetais apresentam distintas, entretanto positiva capacidade em estocar carbono no solo no Brasil e que variáveis como o manejo, a textura, atributos físicos, químicos e biológicos são fatores significativos e podem e influenciam diretamente nos resultados. 


\section{INTRODUÇÃO}

O Brasil se caracteriza por sua grande extensão territorial, apresentando grande biodiversidade ao longo do seu território (Aguiar et al., 2016). Tal diversidade é ameaçada devido a fatores como: o uso em excesso dos recursos naturais, a expansão da fronteira agrícola e da silvicultura, o crescimento urbano e industrial (Peixoto et al., 2016). Atividades antrópicas têm causado a elevação da concentração dos gases de efeito estufa (GEE) na atmosfera (Le Quéré et al., 2018), principalmente devido mudanças causadas na exploração do solo, que alteram a capacidade dos solos em armazenar o $\mathrm{CO}_{2}$ adsorvido pelas plantas (Santana et al., 2019).

Algumas das alternativas para tentar solucionar os problemas causados pelas emissões de GEE é o controle das mesmas (Le Quéré et al., 2018), e a adoção de medidas compensatórias, como aumento de estoque de carbono nos ecossistemas terrestres (Primieri et al., 2017). Uma das formas de aumentar o estoque de carbono está na preservação de florestas nativas; no reflorestamento; na adoção de sistemas integrados de lavoura, pecuária e floresta; e no manejo adequado de pastagens e agricultura (Cook et al., 2016; Vicente et al., 2019; Magalhães et al., 2016), pois essas medidas podem retirar grande quantidade de $\mathrm{CO}_{2}$ da atmosfera pelo processo de fotossíntese (Cassol et al., 2019) e estocar esse carbono na biomassa aérea, subterrânea e principalmente no solo (Zelarayán et al., 2015).

Estudos avaliativos de trabalhos publicados sobre os estoques de carbono sob diferentes usos do solo são de extrema importância na compreensão das mudanças nos níveis de carbono de acordo com o manejo aplicado (Batle-Bayer et al., 2010; Stockmann et al., 2015). Assim a revisão sistemática busca responder a uma questão específica utilizando uma metodologia explícita e sistemática na identificação, seleção e avaliação crítica de estudos a serem incluídos na revisão (Gonçalves, 2019). Em face ao exposto o presente estudo busca revisar publicações sobre os estoques de carbono do solo sob diferentes coberturas vegetais no Brasil publicados nos últimos anos e realizar uma avaliação crítica e sistêmica do assunto.

\section{MATERIAL E MÉTODOS}

Este estudo se constitui em uma revisão sistemática descritiva de caráter analítico de bibliografias que abordam a respeito dos estoques de carbono no solo sob vários tipos de manejo no Brasil. Os dados foram coletados período de 14 a 25 de outubro de 2019, através da base de dados online Scientific Eletronic Library Online (SCIELO) e Science Direct.

Foi utilizado como critério de inclusão artigos atuais publicados nos últimos 10 anos $(2010$ a 2019). Foram incluídos neste estudo artigos que apresentaram descritores como: Carbono orgânico do solo (COS), e Estoques de carbono no solo (ECS) e suas combinações e variantes em inglês para as pesquisas nas bases SCIELO sendo encontrados 96 artigos, onde 18 foram selecionados por se apresentarem de acordo com os objetivos do estudo. A pesquisa na base de dados Science Direct por se tratar de uma base de dados internacional utilizou como descritores: Soils organic carbon in Brazil, Soil carbon stocks in Brazil resultando em 990 artigos de pesquisa dos quais 17 foram selecionados por se encontrarem de acordo com o escopo da pesquisa. Ao final da pesquisa foram selecionados 35 artigos que se correlacionavam com o tema estoques de carbono do solo em diferentes tipos de manejo no Brasil.

Após a seleção dos artigos de acordo com os critérios de inclusão definidos, foram seguidos, os seguintes passos: tradução dos artigos em língua estrangeira, leitura exploratória, leitura seletiva e escolha do material que se adequam aos objetivos e tema do estudo; leitura analítica e análise dos textos, finalizando com a realização de leitura interpretativa (Gonçalves, 2019). Por conseguinte, selecionou-se os temas mais abordados, seguindo a seguinte sequência: ecossistemas brasileiros; clima e solos no Brasil; estoques de carbono no solo em coberturas vegetais nativas; estoques de carbono impostos a mudança no uso do solo.

\section{ECOSSISTEMAS BRASILEIROS}

A vegetação nativa brasileira é bastante diversificada, dividida em 6 biomas, Amazônia, Cerrado, Caatinga, Mata Atlântica, Pantanal e Pampa, ocupando aproximadamente 57,31\% do território nacional, considerando as áreas antrópicas/desmatadas e mudanças no uso do solo (Aguiar et al., 2016).

Amazônia: o bioma amazônico apresenta uma cobertura florestal composta principalmente por floresta ombrófila densa, caracterizada por árvores altas (Cunha et al., 2017) ocupando 3,35 milhões de $\mathrm{km}^{2}, 79,7 \%$ da sua área original (Aguiar et al., 2016), devido a exploração econômica predatória, principalmente pela expansão agrícola e a extração de madeira, entretanto ainda é considerada a maior reserva biológica do planeta (Campos et al., 2016).

Cerrado: o bioma ocupa o planalto central do país e abrangendo mais de 2 milhões de $\mathrm{km}^{2}$, sendo uma das savanas com mais diversidade biológica do planeta (Batlle-Bayer et al., 2010). Suas 
formações vegetais são classificadas como: cerradão (maior porte arbóreo), cerrado, campo sujo e campo limpo (Aguiar et al., 2016). Devido a sua localização, clima e relevo o Cerrado é um grande campo agropecuário, responsável por grande parte da produção de grãos e carne bovina do país (Nunes et al., 2011). Estima-se que haja no cerca de 25,4 milhões de ha de terras convertidas, grande parte em pastagens, com alta aptidão agrícola (Carvalho et al., 2010).

Caatinga: é composta por uma vegetação decidual, espinhosa, também chamada de savanaestépica, compreende aproximadamente $845 \mathrm{mil}$ $\mathrm{km}^{2}$ de área, $10 \%$ do território nacional (Aguiar et al., 2016). A Caatinga é bastante heterogênea, com várias paisagens únicas, com destaque para as ilhas de solos férteis, onde é possível produzir quase todos os alimentos e frutas, essas áreas se localizam próximas às serras, onde as chuvas são mais intensas (Schulz et al., 2016). A Caatinga, porém, sofre alto grau de degradação ambiental, principalmente aos processos de desertificação (Santana et al., 2019). O bioma Caatinga tem aproximadamente $70 \%$ da sua área original alterada podendo citar a exploração predatória de madeira, a substituição da cobertura vegetal por agricultura e fruticultura e o crescimento demográfico da região como as principais causas dessa alteração (Aguiar et al., 2016).

Mata Atlântica: esse bioma e seus ecossistemas associados ocupam $13 \%$ do território brasileiro $(1,1$ milhão de $\mathrm{km}^{2}$ ), visto que foi reduzida a $193 \mathrm{mil}$ $\mathrm{km}^{2}$ atribuído a enorme expansão demográfica na região Sudeste (cerca de 60\%); ao plantio de canade-açucar, café e soja, justificando boa parte do processo de devastação das florestas (Aguiar et al., 2016; Bordonal et al., 2018). O bioma é composto por diversas formações florestais, como floresta ombrófila (densa, mista e aberta), floresta estacional semidecidual e estacional decidual, manguezais, restingas e brejos interioranos no Nordeste (Pegoraro et al., 2016).

Pantanal: se situa nos estados do Mato Grosso e Mato Grosso do Sul, sendo a maior planície inundável do mundo, com relevo plano com altitude média de 150 metros, sendo que no período chuvoso, até $80 \%$ da sua planície é alagada (Aguiar

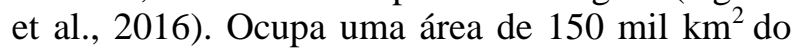
território nacional onde grande parte dessa área é ameaçada devido a agricultura (principalmente soja e cana-de-açucar); exploração mineral e atividade agropecuária (Aguiar et al., 2016; Mello et al., 2015). Sua vegetação é um mosaico de florestas baixas, cerradões, cerrados e campos inundáveis (Cunha et al. 2016).

Pampa: possui uma área aproximada de 176,5 mil km² e ocorre no estado no Rio Grande do Sul apresentando uma vegetação de gramíneas entremeadas por florestas mesófilas, florestas estacionais e florestas subtropicais (Bortoluzzi Castro et al., 2019). Estudos indicam que apenas $1 \%$ do Pampa é protegeido e as principais ameaças a esse bioma é a pecuária, a lavoura de arroz e a expansão do cultivo da soja (Aguiar et al., 2016).

\section{CLIMA E SOLOS}

O Brasil apresenta uma enorme diversidade climática, devido a sua extensão territorial com diferentes tipos de relevo, altitude e dinâmica das massas de ar e das correntes marítimas, fatores esses que influenciam diretamente no clima (Rodrigues Filho et al., 2016). Segundo Dubreuil et al. (2018) os climas que predominam o Brasil são:

- Equatorial: predominante na Amazônia, apresenta quente e úmido e temperaturas médias elevadas de 25 a $27{ }^{\circ} \mathrm{C}$, apresentando chuvas durante todo o ano.

- Tropical: clima presente na porção central do país, com temperaturas que variam de 18 a $28{ }^{\circ} \mathrm{Ce}$ estações bem definidas (uma chuvosa no verão e outra seca no inverno) e uma precipitação média anual de $1500 \mathrm{~mm}$.

- Tropical de Altitude: encontra-se nas partes mais elevadas (acima de 800 metros) no planalto Atlântico do Sudeste estando sob influência da massa de ar tropical atlântica, que provoca chuvas no período do verão. Apresenta baixas temperaturas, entre 18 e $22{ }^{\circ} \mathrm{C}$.

- Tropical Atlântico: compreende parte da faixa litorânea do país e sofre ação direta da massa tropical atlântica. A temperatura varia de 18 a 26 ${ }^{\circ} \mathrm{C}$, sendo que no Nordeste, a maior concentração de chuva ocorre no inverno, e no Sudeste, ocorre no verão. A precipitação média anual fica em torno de $2000 \mathrm{~mm}$.

- Subtropical: ocorre na porção sul do país e é influenciado pela massa polar atlântica, possui temperatura média anual de $18{ }^{\circ} \mathrm{C}$ com chuvas pouco intensas e bem distribuídas, $1000 \mathrm{~mm}$ anuais. O verão é quente $\left(30^{\circ} \mathrm{C}\right)$ e o inverno frio podendo apresentar temperaturas abaixo de $0{ }^{\circ} \mathrm{C}$.

- Semiárido: ocorre na região Nordeste, e caracteriza-se por temperaturas elevadas (média de $27{ }^{\circ} \mathrm{C}$ ) e chuvas escassas e mal distribuídas, em torno de $700 \mathrm{~mm}$ anuais. Há períodos em que a massa equatorial atlântica (superúmida) chega ao litoral norte da região Nordeste e atinge o sertão, causando chuvas intensas no período de fevereiro a abril.

O solo possui funções de destaque nos principais processos biogeoquímicos do planeta, com destaque para o ciclo hidrológico, que contribui para perenização dos cursos de água e controle de 
enchentes (Polidoro et al., 2016), e o ciclo do carbono, fixando esse elemento; reciclando nutrientes; abriga um quarto da biodiversidade do planeta entre outras várias outras funções de destaque (Stockmann et al. 2015).

No Brasil há 13 classes de solos, que subdivididas em subordens que, sucessivamente, se comportam grandes grupos e subgrupos (Polidoro et al., 2016). Os Latossolos, Argissolos e Neossolos são as classes de solo mais representativas em solo brasileiro, ocupando aproximadamente $72 \%$ do território (Tabela 1) (Santos et al., 2018). Latossolos e Argissolos são solos profundos, com alto grau de de intemperismo, ácidos e com baixa fertilidade natural (Santos et al., 2011). Os Neossolos são de média a alta fertilidade, pouco intemperizados e em decorrência disso apresentam baixa profundidade (Santos et al., 2018).

Tabela 01 - Extensão e distribuição das classes de solos no Brasil.

\begin{tabular}{lcc}
\hline Classes & $\begin{array}{c}\text { Área absoluta } \\
\left(\mathbf{k m}^{\mathbf{2}}\right)\end{array}$ & $\begin{array}{c}\text { Área Relativa } \\
(\mathbf{\%})\end{array}$ \\
\hline Argissolos & $2.285 .410,99$ & 26,94 \\
Cambissolos & $446.223,53$ & 5,26 \\
Chernossolos & $37.326,68$ & 0,44 \\
Espodossolos & $167.970,07$ & 1,98 \\
Gleissolos & $397.868,51$ & 4,69 \\
Latossolos & $2.681 .582,83$ & 31,61 \\
Luvissolos & $241.775,10$ & 2,85 \\
Neossolos & $1.123 .193,82$ & 13,24 \\
Nitossolos & $95.861,71$ & 1,13 \\
Organossolos & $2.545,00$ & 0,03 \\
Planossolos & $226.505,10$ & 2,67 \\
Plintossolos & $589.591,92$ & 6,95 \\
Vertissolos & $17.815,01$ & 0,21
\end{tabular}

\begin{tabular}{lcc} 
Outros & $169.666,74$ & 2,00 \\
\hline Total & $8.483 .337,00$ & 100,00 \\
\hline
\end{tabular}

Fonte: Santos et al. (2018).

Os Latossolos geralmente são profundos ( 1 a 2 m) e bastante intemperizados e normalmente de baixa fertilidade (Santos et al., 2018). É uma classe de solo encontrada em áreas planas (principalmente no planalto central brasileiro, bioma Cerrado) entretanto, encontra-se presente em todos os estados do país sendo o solo de maior predominância no Brasil 31,61\% (Batlle-Bayer et al., 2010). Apresenta bons atributos físicos, pois são porosos, permeáveis, com boa drenagem e possui variadas cores (vermelho, amarelo, vermelho amarelado) (Santos et al., 2011), fazendo com que tenham uma alta aptidão agrícola, embora apresentem baixa fertilidade química, as práticas de adubação e correção do solo, os tornam mais produtivos (Magalhães et al., 2016).

\section{ESTOQUES DE CARBONO NO SOLO EM COBERTURAS VEGETAIS NATIVAS}

Os Biomas Amazônico, Cerrado, Mata Atlântica e a Caatinga cobrem cerca de $96 \%$ do território brasileiro (Aguiar et al., 2016), e apesar de sofrerem com a ação humana são responsáveis por grande parte do carbono armazenado nos solos no Brasil (Carvalho et al. 2010). Coberturas florestais não antropizadas proporciona altos estoques de carbono ao solo, devido à alta deposição de nutrientes, matéria orgânica, haja vista o seu grande volume de biomassa aérea, sendo grande reserva de carbono (Zelarayán et al., 2015), que ao longo do tempo é depositada no solo (Primieri et al., 2017).

Tabela 02 - Estoques de Carbono no Solo sob diferentes biomas e coberturas vegetais em Latossolos.

\begin{tabular}{|c|c|c|c|c|c|c|c|c|}
\hline \multirow[b]{2}{*}{$\begin{array}{l}\text { Vegetação } \\
\text { Nativa }\end{array}$} & \multirow[b]{2}{*}{ Bioma } & \multirow[b]{2}{*}{$\begin{array}{l}\text { Classe } \\
\text { de Solo }\end{array}$} & \multirow{2}{*}{$\begin{array}{l}\text { Argila } \\
\left(\text { g.kg-1) }^{-1}\right)\end{array}$} & \multicolumn{4}{|c|}{ ECS (Mg.ha $\left.{ }^{-1}\right)$} & \multirow[b]{2}{*}{ Referências } \\
\hline & & & & $\begin{array}{c}0-20 \\
\text { cm }\end{array}$ & $\begin{array}{c}0-40 \\
\text { cm }\end{array}$ & $\begin{array}{l}0-60 \\
\mathrm{~cm}\end{array}$ & $\begin{array}{c}0-100 \\
\text { cm }\end{array}$ & \\
\hline${ }^{1}$ CESD & & ${ }^{2} \mathrm{~L}$ & 830 & 75,80 & 124,20 & - & 223,60 & Zinn et al. (2014) \\
\hline CSS & & LV & - & 44,72 & - & - & - & $\begin{array}{l}\text { Alcântara Neto et } \\
\text { al. (2011) }\end{array}$ \\
\hline $\mathrm{CD}$ & Cerrado & LV & - & 48,39 & - & - & - & $\begin{array}{l}\text { Alcântara Neto et } \\
\text { al. (2011) }\end{array}$ \\
\hline CS & & LV & - & 31,01 & - & - & - & $\begin{array}{l}\text { Alcântara Neto et } \\
\text { al. (2011) }\end{array}$ \\
\hline CTSD & & LVA & - & 42,10 & - & - & - & $\begin{array}{l}\text { Magalhães et al. } \\
\text { (2016) }\end{array}$ \\
\hline
\end{tabular}


Tabela 02 - Estoques de Carbono no Solo sob diferentes biomas e coberturas vegetais em Latossolos.

\begin{tabular}{|c|c|c|c|c|c|c|c|c|}
\hline \multirow{2}{*}{$\begin{array}{l}\text { Vegetação } \\
\text { Nativa }\end{array}$} & \multirow[b]{2}{*}{ Bioma } & \multirow{2}{*}{$\begin{array}{l}\text { Classe } \\
\text { de Solo }\end{array}$} & \multirow{2}{*}{$\begin{array}{l}\text { Argila } \\
\left(\text { g.kg }^{-1}\right)\end{array}$} & \multicolumn{4}{|c|}{ ECS $\left(\right.$ Mg.ha $\left.^{-1}\right)$} & \multirow[b]{2}{*}{ Referências } \\
\hline & & & & $\begin{array}{c}\mathbf{0 - 2 0} \\
\mathrm{cm}\end{array}$ & $\begin{array}{l}0-40 \\
\text { cm }\end{array}$ & $\begin{array}{l}0-60 \\
\text { cm }\end{array}$ & $\begin{array}{l}\text { 0-100 } \\
\text { cm }\end{array}$ & \\
\hline MMES & \multirow{7}{*}{ Cerrado } & LV & 449 & 43,45 & 68,40 & - & - & Giácomo et al. (2015) \\
\hline CER & & LV & 519 & 39,10 & 66,85 & - & - & Giácomo et al. (2015) \\
\hline CSS & & LV & 294 & 25,50 & 42,56 & - & - & Giácomo et al. (2015) \\
\hline $\mathrm{CE}$ & & LV & 595 & - & 76,50 & & & Coser et al. (2018) \\
\hline CER & & LV & - & 32,70 & 52,92 & 77,88 & 94,30 & Oliveira et al. (2016) \\
\hline $\mathrm{CE}$ & & LV & \multirow{2}{*}{330} & 39,27 & - & - & - & $\begin{array}{l}\text { Rossetti e Centurion, } \\
\text { (2015) }\end{array}$ \\
\hline $\mathrm{CE}$ & & LV & & 36,63 & - & - & - & $\begin{array}{l}\text { Rossetti e Centurion, } \\
\qquad(2015)\end{array}$ \\
\hline FTD & \multirow{2}{*}{ Amazônia } & LVA & 332 & 38,00 & 55,13 & - & - & Araújo et al. (2011) \\
\hline FO & & $\mathrm{L}$ & 466 & 52,50 & - & - & - & Petter et al. (2017) \\
\hline FESDT & \multirow{3}{*}{$\begin{array}{c}\text { Mata } \\
\text { Atlântica }\end{array}$} & LV & 310 & 38,36 & - & - & - & Freitas et al. (2018) \\
\hline FSDS & & LVA & 190 & 31,91 & 51,97 & - & - & Cook et al. (2014) \\
\hline FTSD & & LVA & 310 & 43,04 & 76,17 & 90,45 & - & Pegoraro et al. (2018) \\
\hline CAAD & \multirow[t]{2}{*}{ Caatinga } & $\mathrm{L}$ & - & - & - & - & 72,10 & Santana et al. (2019) \\
\hline CAAA & & $\mathrm{L}$ & - & - & - & - & 63,50 & Santana et al. (2019) \\
\hline
\end{tabular}

${ }^{1}$ CESD: Cerrado Semidecíduo; CSS: Cerrado sensu stricto; CD: Cerrado Denso; CS: Campo Sujo; MMES: Mata Mesofísica; CER: Cerradão; CE: Ceraado; FTD: Floresta Tropical Densa; FO: Floresta Ombrófila; FESDT: Floresta Estacional semidecídua tropical; FSDS: Floresta Semidecídua sazonal; FTSD: Floresta Tropical seca decídua; CAAD: Caatinga Densa; CAAA: Caatinga Aberta.

${ }^{2}$ L: Latossolo; LV: Latossolo Vermelho; LVA: Latossolo Vermelho Amarelo distrófico.

\section{ESTOQUES DE CARBONO NO SOLO IMPOSTOS A MUDANÇA NO USO DO SOLO}

A agricultura é um grande impulsionador da economia brasileira (Maia et al., 2010), visto isso a conversão de áreas naturais em terras agrícolas cresce em escala proporcional, com números atuais de 62,1 milhões de ha (IBGE, 2018).

A produção agrícola brasileira se concentra principalmente nas regiões Centro-Oeste e Sul ocupando $73,4 \%$ da área produtiva do país (Tabela 3) (IBGE, 2018).

Em frente a isso vê-se a necessidade de avaliar os estoques de carbono no solo em cultivos agrícolas (Stockmann et al., 2015), visto a grande área ocupada pela agricultura no Brasil. A agricultura apresenta variações nos ECS de acordo com o tipo de manejo adotado. Sistemas conservacionistas apresentam uma melhor capacidade em estocar carbono devido a uma menor intervenção nos processos de preparo e plantio do solo em relação ao cultivo convencional (Bordonal et al., 2018; Campos et al., 2011).
Estudos de Coser et al. (2018) avalia que em curto prazo cultivos conservacionistas possuem uma grande capacidade em recuperar o carbono perdido com a implantação de culturas agrícola. Esses sistemas como o Plantio Direto (PD) representam um pouco mais de $50 \%$ das terras agrícolas no país (Rossetti e Centurion, 2015).

Tabela 03 - Área de cereais, leguminosas e oleaginosas nas regiões do Brasil.

\begin{tabular}{lcc}
\hline Região & $\begin{array}{c}\text { Área Total } \\
\text { (ha) }\end{array}$ & $\begin{array}{c}\text { Particul. } \\
(\%)\end{array}$ \\
\hline Norte & 2.882 .636 & 4,64 \\
Nordeste & 8.146 .493 & 13,11 \\
Sudeste & 5.502 .489 & 8,86 \\
Sul & 19.566 .180 & 31,49 \\
Centro-Oeste & 26.038 .110 & 41,91 \\
\hline Brasil & 62.135 .908 & 100 \\
\hline
\end{tabular}

Fonte: (IBGE, 2018) 
Tabela 04 - Estoques de carbono no solo sob diferentes tipos de cultivos culturais no Brasil.

\begin{tabular}{|c|c|c|c|c|c|c|c|}
\hline \multirow{2}{*}{ Tipo de Cultivo } & \multirow{2}{*}{$\begin{array}{l}\text { Classe } \\
\text { de Solo }\end{array}$} & \multirow{2}{*}{$\begin{array}{c}\text { Argila } \\
\left(\mathrm{g} \mathrm{kg}^{-1}\right)\end{array}$} & \multicolumn{3}{|c|}{ ECS $\left(\right.$ Mg.ha $\left.^{-1}\right)$} & \multirow{2}{*}{$\begin{array}{l}\text { Tempo } \\
\text { (anos) }\end{array}$} & \multirow{2}{*}{ Referências } \\
\hline & & & $0-20 \mathrm{~cm}$ & $0-30 \mathrm{~cm}$ & $0-40 \mathrm{~cm}$ & & \\
\hline${ }^{1} \mathrm{PD}$ Soja & ${ }^{2} \mathrm{~L}$ & 481 & 40,50 & - & - & 5 & Peter et al. (2017) \\
\hline PD soja & LV & 330 & 35,93 & 50,55 & - & 9 & Rossetti e Centurion (2015) \\
\hline PC soja & LV & 330 & 26,30 & 36,23 & - & 9 & Rossetti e Centurion (2015) \\
\hline PD milheto & LV & 571 & 36,55 & - & - & 11 & Nunes et al. (2011) \\
\hline PC milheto & LV & 571 & 34,36 & - & - & 11 & Nunes et al. (2011) \\
\hline PD trigo & LV & 510 & 55,03 & 74,90 & - & 9 & Campos et al. (2011) \\
\hline PC trigo & $\mathrm{LV}$ & 520 & 53,98 & 74,86 & - & 9 & Campos et al. (2011) \\
\hline PD milho/sorgo & $\mathrm{L}$ & - & - & 57,97 & - & 20 & Maia et al. (2010) \\
\hline PC arroz/soja & LVA & 300 & 40,65 & - & - & 9 & Magalhães et al. (2016) \\
\hline PC MCC & $\mathrm{L}$ & 295 & 28,92 & - & - & 9 & Bordonal et al. (2017) \\
\hline PC fumo 2 & RL & 161 & 59,55 & - & - & 2 & Stürmer et al. (2011) \\
\hline PC fumo 15 & $\mathrm{RL}$ & 110 & 16,49 & - & - & 15 & Stürmer et al. (2011) \\
\hline Cana-de-açúcar & $\mathrm{L}$ & 288 & 30,67 & - & - & 9 & Bordonal et al. (2017) \\
\hline Cana-de-açúcar & $\mathrm{LV}$ & 303 & 25,77 & - & - & 60 & Freitas et al. (2018) \\
\hline Cana-de-açúcar & LV & - & - & 38,15 & - & 6 & Oliveira et al. (2016) \\
\hline Cana-de-açúcar & $\mathrm{N}$ & - & - & 40,10 & - & 5 & Oliveira et al. (2016) \\
\hline Cana-de-açúcar & $\mathrm{P}$ & - & - & 60,50 & - & 25 & Oliveira et al. (2016) \\
\hline Cana-de-açúcar & LVA & 280 & 39,93 & - & 70,88 & 2 & Pegoraro et al. (2018) \\
\hline Cana-de-açúcar CMan & $\mathrm{L}$ & 687 & 88,40 & - & 179,80 & 40 & Luca et al. (2018) \\
\hline Cana-de-açúcar CMan & $\mathrm{P}$ & 750 & 45,70 & - & 91,50 & 40 & Luca et al. (2018) \\
\hline Cana-de-açúcar CMan & RQ & 865 & 39,70 & - & 79,80 & 40 & Luca et al. (2018) \\
\hline Cana-de-açúcar CMec & $\mathrm{L}$ & 660 & 100,00 & - & 203,00 & 40 & Luca et al. (2018) \\
\hline Cana-de-açúcar CMec & $\mathrm{P}$ & 760 & 50,40 & - & 102,30 & 40 & Luca et al. (2018) \\
\hline Cana-de-açúcar CMec & RQ & 840 & 47,10 & - & 89,50 & 40 & Luca et al. (2018) \\
\hline
\end{tabular}

${ }^{1}$ PD: Plantio Direto; PC: Plantio Convencional; MCC: Milho/Cebola/Cenoura; CMan: Colheita Manual; CMec: Colheita Mecanizada.

${ }^{2}$ L: Latossolo; LV: Latossolo Vermelho; LVA: Latossolo Vermelho Amarelo; RL: Neossolo Litólico; N: Nitossolo; P: Argissolo; RQ: Neossolo Quartzarênico.

Apesar de menos eficiente na captação de carbono o Plantio Convencional (PC) ainda tem espaço no cenário nacional, devido a uma maior produtividade, em comparação ao $\mathrm{PD}$, apesar de não apresentar diferenças significativas na produção, o PC apresenta um custo mais alto em comparação ao PD (Rossetti e Centurion, 2015).

Além do manejo adotado, fatores como o tipo de solo, a textura (teor de argila) e a mineralogia são fatores significativos que alteram os ECS (Oliveira et al., 2016). Estudos de (Stürmer et al., 2011) em um PC de fumo com 15 anos de idade, relatou grande deficiência em estocar carbono no solo do plantio, devido ao baixo teor de argila (em média $110 \mathrm{~g} \mathrm{~kg}^{-1}$ ) aliado ao uso inadequado e o intenso revolvimento do solo (arações, gradagens e capinas) se comparado ao solo em vegetação nativa onde a perturbação do solo é baixa ou nula. Já cultivos em solos que apresentam teores de argila acima de $300 \mathrm{~g} \mathrm{~kg}^{-1}$ tendem a ter um melhor desempenho em estocar carbono (Luca et al., 2018). Segundo Nunes et al. (2011) e Campos et al. (2011) muito devido ao alto teor de argila presente no solo $\left(571 \mathrm{~g} \mathrm{~kg}^{-1}\right.$ e $510 \mathrm{~g} \mathrm{~kg}^{-1}$ respectivamente), $\mathrm{PC}$ e $\mathrm{PD}$ de milheto e trigo não apresentaram diferenças significativas nos seus respectivos ECS.

Em região de cerrado, estudos de Maia et al. (2010) relatam que o tipo de solo não é um fator significativo nas diferenças nos ECS, visto que a prática agrícola se apresenta como o fator mais significativo na definição dos ECS. Ainda segundo o autor, nas regiões de floresta mais densa onde houve PD teve pouco ou nenhum impacto no ECS após a conversão de florestas nativas, com o 
armazenamento de carbono aumentando após 20 anos implantação da agricultura.

A avaliação dos estoques de carbono no solo (ECS) em diferentes estágios sucessionais em variados tipos de uso e manejo do solo mostra que ao longo dos anos o carbono perdido pela implantação de diferentes manejos pode ser recuperado e até aumentado em relação a áreas nativas não alteradas (Gatto et al., 2010) (Tabela 5).

Tabela 05 - Estoques de carbono no solo em diferentes profundidades sob vegetação nativa impostas a mudanças no uso do solo no Brasil.

\begin{tabular}{|c|c|c|c|c|c|c|c|c|c|}
\hline \multirow[b]{2}{*}{ Referência } & \multirow[b]{2}{*}{ Plantio } & \multirow{2}{*}{$\begin{array}{c}\text { Classe } \\
\text { de } \\
\text { Solo }\end{array}$} & \multirow{2}{*}{$\begin{array}{c}\text { Argila } \\
\left(\text { g.kg-1 }^{-1}\right)\end{array}$} & \multirow{2}{*}{$\begin{array}{l}\text { Profund. } \\
\quad \text { (cm) }\end{array}$} & \multicolumn{2}{|c|}{ Vegetação Nativa } & \multicolumn{2}{|c|}{ Novo uso do solo } & \multirow{2}{*}{$\begin{array}{l}\text { Tempo } \\
\text { (anos) }\end{array}$} \\
\hline & & & & & Tipo & $\begin{array}{c}\text { ECS } \\
\left(\text { Mg.ha' }^{-1}\right)\end{array}$ & Tipo & $\begin{array}{c}\text { ECS } \\
\left(\text { Mg.ha' }^{-1}\right)\end{array}$ & \\
\hline Alcântara Neto et al. (2011) & \multirow{7}{*}{ Floresta } & $\mathrm{LV}^{1}$ & - & $0-20$ & $\mathrm{CD}^{2}$ & 48,40 & $\operatorname{Re}^{3}$ & 33,00 & 7 \\
\hline Vicente et al. (2019) & & PVA & 566 & $0-100$ & FS & 168,30 & $\operatorname{Re}$ & 160,70 & 5 \\
\hline Pegoraro et al. (2014) & & PA & 270 & $0-100$ & FOD & 100,00 & $\operatorname{Re}$ & 83,00 & 24 \\
\hline Pegoraro et al. (2014) & & PA & 280 & $0-100$ & FOD & 100,00 & $\mathrm{Ra}$ & 94,00 & 14 \\
\hline Cassol et al. (2019) & & $\mathrm{CH}$ & 178 & $0-40$ & PN & 109,00 & $\mathrm{Rp}$ & 144,10 & 21 \\
\hline Frazão et al. (2014) & & LA & 210 & $0-30$ & FA & 48,50 & RfD & 61,10 & 34 \\
\hline Maia et al. (2010) & & $\mathrm{L}$ & - & $0-30$ & FAA & 46,80 & RfC & 51,50 & 9 \\
\hline Araújo et al. (2011) & \multirow{5}{*}{ Pastagem } & PVA & 265 & $0-40$ & FTA & 33,30 & Bbri. & 51,90 & 10 \\
\hline Magalhães et al. (2016) & & LVA & - & $0-20$ & CSS & 42,10 & Bbri. & 54,50 & 13 \\
\hline Santos et al. (2019) & & PA & 400 & $0-30$ & FA & 49,30 & Bbri. & 66,60 & 16 \\
\hline Vicente et al. (2019) & & PVA & 520 & $0-40$ & FS & 93,10 & Bdec. & 91,20 & 50 \\
\hline Pegoraro et al. (2018) & & LVA & 310 & $0-20$ & FTSD & 43,04 & Bdec. & 37,43 & 3 \\
\hline Rossetti \& Centurion (2015) & \multirow{4}{*}{ Agricultura } & LV & 330 & $0-30$ & $\mathrm{CE}$ & 54,50 & PD & 50,60 & 9 \\
\hline Rossetti \& Centurion (2015) & & LV & 330 & $0-30$ & $\mathrm{CE}$ & 49,10 & PD & 44,10 & 10 \\
\hline Maia et al. (2010) & & $\mathrm{L}$ & - & $0-30$ & CSS & 61,40 & $\mathrm{PC}$ & 58,00 & 20 \\
\hline Magalhães et al. (2016) & & LVA & 300 & $0-20$ & CSS & 42,10 & $\mathrm{PC}$ & 40,65 & 9 \\
\hline
\end{tabular}

${ }^{1}$ L: Latossolo; LV: Latossolo Vermelho; LA: Latossolo Amarelo; LVA: Latossolo Vermelho Amarelo distrófico; PA: Argissolo Amarelo; PVA: Argissolo Vermelho Amarelo; CH: Cambissolo Húmico.

${ }^{2}$ CD: Cerrado Denso; FS: Floresta Secundária; FOD: Floresta Ombrófila Densa; PN: Pastagem Natural; FA: Floresta Altântica; FAA: Floresta Amazônica Aberta; FTA: Floresta Tropical Aberta; CE: Cerrado; CSS: Cerrado sensu stricto; FTSD: Floresta Tropical seca decídua.

${ }^{3}$ Re: Eucalyptus; Ra: Acacia Mangium; Rp: Pinus taeda; RfD: Dendêzeiro; RfC: Café Arábica; Bbri: Brachiaria Brizantha; Bdec: Brachiaria Decubens; PD: Plantio Direto; PC: Plantio Convencional.

Ao longo dos anos, solos de áreas reflorestadas mostram uma capacidade maior em recuperar o carbono perdido na área desmatada (Frazão et al., 2014). Plantios florestais de longa rotação apresentam maior capacidade em estocar carbono pois, ao longo do tempo de maturação da floresta há uma maior deposição de serapilheira o que gera uma barreira física e auxiliando nas propriedades do solo, e no potencial em estocar carbono no solo (Cassol et al., 2019; Pulrolnik et al., 2009). Cultivos florestais de longa rotação também apresentam um maior aporte de carbono nas substâncias húmicas do solo, muito devido as baixas taxas de mineralização da MOS pelo não revolvimento e exposição do solo causada por atividades de colheita e preparo da área para novas plantações longa rotação (Pegoraro et al., 2014). Para Vicente et al. (2019) os teores de argila presente nos microagregados do solo, são os principais responsáveis a alta capacidade em estocar carbono no solo em plantios florestais como de Eucalyptus. Outra teoria para os altos níveis de carbono no solo em sistemas florestais se dá a maior presença de biomassa radicular principalmente nas camadas superficiais do solo (Frazão et al., 2014). Os cultivos mais longos apresentam ECS mais altos em relação à vegetação nativa. No entanto, resultados estimam que os sistemas de culturas perenes retêm cerca de $98 \%$ da quantidade de COS encontrada sob vegetação nativa (Maia et al., 2010).

Estoques de carbono em pastagens cultivadas apresentam uma grande capacidade em estocar carbono no solo nas camadas superficiais, devido 
ao acúmulo de matéria orgânica à superfície e em profundidade (Campos et al., 2016), assim os maiores estoques de carbono no solo encontram-se em ambientes de pastagens mais antigas (Araújo et al., 2011). As pastagens, geralmente ocupam áreas de solo com características físicas semelhantes, com predominância de solos com aproximadamente $40 \%$ de argila (Freitas et al., 2018). Solos argilosos apresentam melhor capacidade em estocar carbono sob pastagens em comparações a mata nativas, devido a correlação de matéria orgânica com a superfície da fração de argila, que auxilia na floculação e na formação de agregados, protegendo a matéria orgânica da degradação (Alcântara Neto et al., 2011). Pastagens mais jovens tendem a apresentar ECS menores devido ao pouco aporte de matéria orgânica nos primeiros anos de implantação, além das perdas de C por intermédio das queimadas (Araújo et al., 2011).

No Brasil grande parte da sua área é ocupada por pastagens cultivadas para pecuária, agricultura e silvicultura, além de sistemas integrados como as agroflorestas (Carvalho et al., 2010). As mudanças no uso do solo quando bem manejadas apresentam boa capacidade em estocar carbono no solo (Campos et al., 2016; Cassol et al., 2019; Vicente et al., 2019; Rossetti \& Centurion, 2015), entretanto nos trabalhos analisados, os valores médios dos ECS se encontram abaixo da média se comparada a vegetação nativa (27,9\% menor), considerando os primeiros $20 \mathrm{~cm}$ do solo (Tabela 6).

Tabela 06 - Valores médios dos estoques de carbono no solo na profundidade de $0-20 \mathrm{~cm}$ em diferentes coberturas vegetais no Brasil.

\begin{tabular}{lc}
\hline \multirow{2}{*}{ Cobertura Vegetal } & ECS $\left(\mathbf{M g . h \mathbf { h } ^ { - 1 }}\right)$ \\
\cline { 2 - 2 } & $\mathbf{0 - 2 0} \mathbf{~ c m}$ \\
\hline Floresta Nativa & $64,77 \mathrm{a}$ \\
Floresta Plantada & $45,93 \mathrm{c}$ \\
Pastagem & $49,40 \mathrm{~b}$ \\
Agricultura & $44,80 \mathrm{c}$ \\
\hline
\end{tabular}

Para Bordonal et al. (2017) os valores de ECS para pastagens e a agricultura são bem maiores considerando a profundidade de $0-100 \mathrm{~cm}$, visto que esses manejos geram uma maior intervenção nas camadas superficiais do solo muito atribuído ao seu sistema radicular presente na camada superficial do solo, não interferindo nos estoques preservados nas camadas mais profundas.

Em geral, mudanças no uso do solo geram perdas significativas dos ECS em comparação a ambientes não alterados (Bordonal et al., 2017), em contrapartida, ao longo dos anos sistemas conservacionistas tendem a recuperar e superar o carbono perdido em comparação a matas nativas (Cassol et al., 2019; Santos et al., 2019b).

\section{CONCLUSÕES}

Visto a intensa mudança no uso do solo, principalmente em solos sob Cerrado, percebeu-se que diferentes coberturas vegetais apresentam distintas, entretanto positiva capacidade em estocar carbono no solo no Brasil. As diferenças significativas encontradas nas médias avaliadas nas diferentes coberturas vegetais foram de $31,61 \%$ para a vegetação nativa, $22,42 \%$ para florestas plantadas, $24,11 \%$ para pastagens e $21,86 \%$ para a agricultura. As coberturas vegetais variaram de acordo aos dados analisados, visto que, o manejo, a textura, atributos físicos, químicos e biológicos são fatores significativos e influenciam diretamente nos resultados de uma pesquisa.

Devido à grande extensão territorial, variedade ecossistêmica assim como a variedade de solos que o Brasil apresenta, estudos mais detalhados e uma melhor uniformização na análise dos dados pode ser proposta, com o apoio de novas ferramentas como a geoestatística, em vista a ter uma mais ampla mensuração dos estoques de carbono totais no Brasil.

\section{REFERÊNCIAS BIBLIOGRÁFICAS}

Aguiar S, Santos IS, Arêdes N, Silva S. Biome-networks: information and communication for sociopolitical action in eco-regions. Ambiente \& sociedade, v. 19, n. 3, p. 231 248, 2016.

Alcântara Neto F, Leite LFC, Arnhold E, Maciel GA, Carneiro RFV. Compartimentos de carbono em Latossolo vermelho sob cultivo de eucalipto e fitofisionomias de cerrado. Revista Brasileira de Ciencia do Solo, v. 35, p. 849856, 2011.

Araújo EA, Ker JC, Mendonça ES, Silva IR, Oliveira EK. Impacto da conversão floresta - pastagem nos estoques e na dinâmica do carbono e substâncias húmicas do solo no bioma Amazônico. Acta Amazonica, v. 41, p. 103-114, 2011.

Batlle-Bayer L, Batjes NH, Bindraban PS. Changes in organic carbon stocks upon land use conversion in the Brazilian Cerrado: A review. Agriculture, Ecosystems and Environment, v. 137, p. 47-58, 2010.

Bordonal RO, Lal R, Ronquim CC, Figueiredo EB, Carvalho JLN, Maldonado Jr. W et al. Changes in quantity and quality of soil carbon due to the land-use conversion to sugarcane (Saccharum officinarum) plantation in southern Brazil. Agriculture, Ecosystems and Environment, v. 240, p. 54-65, 2017. 
Bordonal RO, Menandro LMS, Barbosa LC, Lal R, Milori DMBP, Kolln OT et al. Sugarcane yield and soil carbon response to straw removal in south-central Brazil. Geoderma, v. 328, p. 79-90, 2018.

Bortoluzzi Castro LR, Carvalho AV, Soares JR, Castro Pessano, EF. Os Biomas Brasileiros nos livros didáticos de Ciências: Um olhar ao Pampa Gaúcho. Revista Electrónica Investigación en Educación en Ciencias, v. 14, n. 1, p. 3849, 2019.

Campos MCC, Soares MDR, Nascimento MF, Silva DMP. Estoque de carbono no solo e agregados em Cambissolo sob diferentes manejos no sul do Amazonas. Revista Ambiente e Agua, v. 11, p. 339-349, 2016.

Campos, B-HC, Amado TJC, Bayer C, Nicoloso RS, Fiorin JE. Carbon stock and its compartments in a subtropical oxisol under long-term tillage and crop rotation systems. Revista Brasileira de Ciência do Solo, v. 35, p. 805-817, 2011.

Carvalho JLN, Avanzi JC, Silva MLN, Mello CR, Cerri CEP. Potencial de sequestro de carbono em diferentes biomas do Brasil. Revista Brasileira de Ciencia do Solo, v. 34, p. 277 289, 2010.

Cassol PC, Fachini L, Mafra ÁL, Brand MA, Simonete M, Coimbra JLM. Alterações no carbono orgânico do solo de campo natural submetido ao plantio de Pinus taeda em três idades. Ciência Florestal, v. 29, p. 545-558, 2019.

Cook RL, Binkley D, Mendes JCT, Stape JL. Soil carbon stocks and forest biomass following conversion of pasture to broadleaf and conifer plantations in southeastern Brazil. Forest Ecology and Management, v. 324, p. 37-45, 2014.

Cook RL, Binkley D, Stape JL. Eucalyptus plantation effects on soil carbon after 20 years and three rotations in Brazil. Forest Ecology and Management, v. 359, p. 92-98 2016.

Coser TR, Figueiredo CC, Jovanovic B, Moreira TN, Leite GG, Salomon SL et al. Short-term buildup of carbon from a low-productivity pastureland to an agrisilviculture system in the Brazilian savannah. Agricultural Systems, v. 166, p. 184-195, 2018.

Cunha CN, Girard P, Nunes GM, Arieira J, Penha J, Junk WJ. Pantanal: A identidade de uma grande área úmida. In: Peixoto AL, Luz JRP, Brito MA. Conhecendo a biodiversidade. Brasília: MCTIC, CNPq, PPBio, 196p. 2016.

Cunha JM, Gaio DC, Campos MCC, Soares MDR, Silva DMP, Lima AFL. Atributos físicos e estoque de carbono do solo em áreas de Terra Preta Arqueológica da Amazônia. Revista Ambiente e Agua, v. 12, p. 263-281, 2017.

Dubreuil V, Fante KP, Planchon O, Sant'anna Neto JL. Os tipos de climas anuais no Brasil: uma aplicação da classificação de Köppen de 1961 a 2015. Confins, v. 37, oct, 2018. Disponível em: <http://journals.openedition.org/confins/15738>. Acesso em : 07 Nov. 2019.

Frazão LA, Paustian K, Cerri CEP, Cerri CC Soil carbon stocks under oil palm plantations in Bahia State, Brazil. Biomass and Bioenergy, v. 62, p. 1-7, 2014.

Freitas L, Oliveira IA, Casagrande JC, Silva LS, Campos MCC. Estoque de carbono de Latossolos em sistemas de manejo natural e alterado. Ciencia Florestal, v. 28, p. 228$239,2018$.
Gatto A, Barros NF, Novais RF, Silva IR, Leite HG, Leite FP et al. Estoques de carbono no solo e na biomassa em plantações de eucalipto. Revista Brasileira de Ciencia do Solo, v. 34, p. 1069-1079, 2010.

Giácomo RG, Pereira MG, Guareschi RF, Machado DL. Atributos químicos e físicos do solo, estoques de carbono e nitrogênio e frações húmicas em diferentes formações vegetais. Ciencia Florestal, v. 25, p. 617-631, 2015.

Gonçalves MJR. Como escrever um artigo de revisão de literatura. Revista JRG de Estudos Acadêmicos, v. 2, n. 5, p. 29-55, ago. 2019.

Instituto Brasileiro de Geografia e Estatística (IBGE). Mapa de vegetação brasileira. 2018. Disponível em: <http://geoftp.ibge.gov.br/informacoes_ambientais/vegetacao/vetores/escala_250_mil/brasil> Acesso em: 01 Nov. 2019.

Le Quéré C, Andrew RM, Friedlingstein P, Sitch S, Hauck J, Pongratz J et al. Global Carbon Budget 2018. Earth System Science Data, v. 10, p. 2141-2194, 2018.

Luca EF, Chaplot V, Mutema M, Feller C, Ferreira ML, Cerri $\mathrm{CC}$ et al. Effect of conversion from sugarcane preharvest burning to residues green-trashing on SOC stocks and soil fertility status: Results from different soil conditions in Brazil. Geoderma, v. 310, v. 238-248, 2018.

Magalhães SSA, Ramos FT, Weber OLS. Estoques de carbono em Latossolo após trinta e oito anos sob diferentes sistemas de cultivo. Revista Brasileira de Engenharia Agricola e Ambiental, v. 20, p. 85-91, 2016.

Maia SMF, Ogle SM, Cerri CC, Cerri CEP. Changes in soil organic carbon storage under different agricultural management systems in the Southwest Amazon Region of Brazil. Soil and Tillage Research, v. 106, p. 177-184, 2010.

Mello JM, Couto EG, Amorim RSS, Chig LA, Johnson MS, Lobo FA. Dinâmica dos atributos físico-químicos e variação sazonal dos estoques de carbono no solo em diferentes fitofisionomias do pantanal norte Mato-Grossense. Revista Arvore, v. 39, p. 325-336, 2015.

Nunes RS, Lopes AAC, Sousa DMG, Mendes IC. Sistemas de manejo e os estoques de carbono e nitrogênio em Latossolo de cerrado com a sucessão soja-milho. Revista Brasileira de Ciencia do Solo, v. 35, p. 1407-1419, 2011.

Oliveira DMS, Paustian K, Davies CA, Cherubin MR, Franco ALC, Cerri CC et al. Soil carbon changes in areas undergoing expansion of sugarcane into pastures in southcentral Brazil. Agriculture, Ecosystems and Environment, v. 228 , p. $38-48,2016$.

Pegoraro RF, Moreira CG, Dias DG, Silveira TC. Carbon and nitrogen stocks in the soil and humic substances of agricultural crops in the semi-arid region. Revista Ciencia Agronomica, v. 49, p. 574-583, 2018.

Pegoraro RF, Silva IR, Novais RF, Barros NF, Cantarutti RB, Fonseca S. Carbon and nitrogen stocks in the alfisol submitted to the Eucalyptus urograndis monoculture and Acacia mangium rotation. Ciencia Florestal, v. 24, p. 933-943, 2014.

Peixoto AL, Luz JRP, Brito MA. Conhecendo a biodiversidade. Brasília: MCTIC, CNPq, PPBio, 196p. 2016.

Petter FA, Lima LB, Morais LA, Tavanti RFR, Nunes ME, Freddi OS et al. Carbon stocks in oxisols under agriculture 
and forest in the southern Amazon of Brazil. Geoderma Regional, v. 11, v. 53-61, 2017.

Polidoro JC, Mendonça-Santos ML, Lumbreras JF, Coelho MR, Carvalho Filho A, Motta PEF et al. Programa Nacional de Solos do Brasil (PronaSolos). Dados eletrônicos. Rio de Janeiro : Embrapa Solos, 53p. 2016.

Primieri S, Muniz AW, Lisboa HM. Dinâmica do Carbono no Solo em Ecossistemas Nativos e Plantações Florestais em Santa Catarina. Floresta e Ambiente, v. 24, e00110314, 2017.

Rodrigues Filho S, Lindoso DP, Bursztyn M, Nascimento CG. O clima em transe: políticas de mitigação e adaptação no Brasil. Revista Brasileira de Climatologia, ano 12, v. 19, p. 74-91, 2016.

Rossetti KV, Centurion JF. Estoque de carbono e atributos físicos de um Latossolo em cronossequência sob diferentes manejos. Revista Brasileira de Engenharia Agricola e Ambiental, v. 19, p. 252-258, 2015.

Santana MS, Sampaio EVSB, Giongo V, Menezes RSC, Jesus KN, Albuquerque ERGM et al. Carbon and nitrogen stocks of soils under different land uses in Pernambuco state, Brazil. Geoderma Regional, v. 16, e00205, 2019.

Santos CA, Rezende CP, Pinheiro EFM, Pereira JM, Alves BJR, Urquiaga $S$ et al. Changes in soil carbon stocks after land-use change from native vegetation to pastures in the Atlantic forest region of Brazil. Geoderma, v. 337, p. 394401, 2019.

Santos HG Carvalho Junior W, Dart RO, Aglio MLD, Sousa JS, Pares JG et al. O novo mapa de solos do Brasil: legenda atualizada. Dados eletrônicos. - Rio de Janeiro : Embrapa Solos, 67p. 2011.

Santos HG, Jacomine PKT, dos Anjos LHC, Oliveira VÁ, Lumbreras JF, Coelho MR et al. Sistema Brasileiro de Classificação de Solos. 5. ed., rev. e ampl. - Brasília, DF : Embrapa, 2018.

Santos LR, Lima AMN, Cunha JC, Rogrigues MS, Soares BEM, Santos LPA et al. Does irrigated mango cultivation alter organic carbon stocks under fragile soils in semiarid climate? Scientia Horticulturae, v. 255, p. 121-127, 2019 b.

Schulz K, Voigt K, Beusch C, Almeida-Cortez JS, Kowarik I, Walz A et al. Grazing deteriorates the soil carbon stocks of Caatinga forest ecosystems in Brazil. Forest Ecology and Management, v. 367, p. 62-70, 2016.

Stockmann U, Padarian J, McBratney A, Minasny B, Broginez D, Montanarella L et al. Global soil organic carbon assessment. Global Food Security, v. 6, p. 9-16, 2015.

Stürmer SLK, Rossato OB, Copetti ACC, Santos DR, Calegari A, Brum B. Variações nos teores de carbono orgânico em função do desmatamento e revegetação natural do solo. Ciencia Florestal, v. 21, p. 241-250, 2011.

Vicente LC, Gama-Rodrigues EF, Gama-Rodrigues AC. Organic carbon within soil aggregates under forestry systems and pasture in a southeast region of Brazil. Catena v. 182, e104139, 2019.

Zelarayán, MLC, Celentano D, Oliveira EC, Triana SP, Sodré DN, Muchavisoy KHM et al. Impacto da degradação sobre o estoque total de carbono de forestas ripárias na
Amazônia Oriental, Brasil. Acta Amazonica v. 45, p. 271$282,2015$.

Zinn YL, Guerra AR, Silva CA, Faria JÁ, Silva TAC. Soil organic carbon and morphology as affected by pine plantation establishment in Minas Gerais, Brazil. Forest Ecology and Management, v. 318, p. 261-269, 2014. 